\title{
Sub-micron sized saccharide fibres via electrospinning
}

DOI 10.1515/esp-2016-0001

Received Jul 7, 2015; accepted Sep 14, 2015

\begin{abstract}
In this work, the production of continuous submicron diameter saccharide fibres is shown to be possible using the electrospinning process. The mechanism for the formation of electrospun polymer fibres is usually attributed to the physical entanglement of long molecular chains. The ability to electrospin continuous fibre from a low molecular weight saccharides was an unexpected phenomenon. The formation of sub-micron diameter "sugar syrup" fibres was observed in situ using highspeed video. The trajectory of the electrospun saccharide fibre was observed to follow that typical of electrospun polymers. Based on initial food grade glucose syrup tests, various solutions based on combinations of syrup components, i.e. mono-, di- and tri-saccharides, were investigated to map out materials and electrospinning conditions that would lead to the formation of fibre. This work demonstrated that sucrose exhibits the highest propensity for fibre formation during electrospinning amongst the various types of saccharide solutions studied. The possibility of electrospinning low molecular weight saccharides into sub-micron fibres has implications for the electrospinability of supramolecular polymers and other biomaterials.
\end{abstract}

Keywords: electrospinning; saccharides; carbohydrates

Pablo G. T. Lepe: MacDiarmid Institute for Advanced Materials and Nanotechnology, Department of Mechanical Engineering, University of Canterbury, Private Bag 4800, Christchurch 8140, New Zealand

Nick Tucker, Lyall Simmons: Biomaterials Engineering and Chemistry, Plant and Food Research Limited, Private Bag 4704, Lincoln 7608, New Zealand

Andrew J. A. Watson, Antony J. Fairbanks: Department of Chemistry, University of Canterbury, Private Bag 4800, Christchurch 8140, New Zealand

*Corresponding Author: Mark P. Staiger: MacDiarmid Institute for Advanced Materials and Nanotechnology, Department of Mechanical Engineering, University of Canterbury, Private Bag 4800, Christchurch 8140, New Zealand; Email: mark.staiger@canterbury.ac.nz; Tel: (+64) (3) 364 2987; Fax: (+64) (3) 3642078

(cc) BY-NC-ND This work is licensed under the Creative Commons Attribution-NonCommercial-NoDerivs 3.0 License.

\section{Introduction}

Electrospinning is a simple method for producing continuous polymer fibres with diameters in the sub-micron range [1-3]. During electrospinning a high voltage is applied to a small droplet of polymer solution (or melt), generating an electric field that stretches the droplet into a conical shape ( $a k a$ the Taylor cone). The build-up of electrical charge due to the electric field overcomes the surface tension of the droplet, and a jet of polymer solution is emitted from the tip of the Taylor cone that may be collected at the nearest earthed object whereupon the jet is electrically discharged. The polymer solution is drawn into a fibre during flight if it has the appropriate electro-viscoelastic properties prior to reaching the collector $[1,3,4]$.

The electrospinning of biopolymers such as chitosan polysaccharides [5-10], cellulose compounds [11-14], and proteins like collagen [15-18], zein [19-24], bovine serum albumin [25] and others [25-30] is well described in the literature. Electrospun polysaccharides [31, 32] and other bio-polymer based nanofibres show significant potential for a variety of biomedical applications [33-35] such as tissue engineering, wound dressing and cosmetics [15, 34, $36,37]$. While oligosaccharides and polysaccharides are known to form fibres by electrospinning [15, 31, 32, 38], the electrospinning of mono- or disaccharide-based solutions into fibres has not been reported yet. However, there are reports of the potential applications that low molecular weight saccharides could have in medicine, biology and microbiology [39] as well as in electrochemistry, and nanotechnology [40]. Hence, the electrospinning of saccharides could lead to the development of new devices for biosensing and drug-delivery [32, 38, 41, 42].

The present work demonstrates that short chain oligosaccharides (e.g. di-saccharides) are able to be electrospun into sub-micron sized fibres. Based on polymer chain entanglement theory, it has been postulated that at least 2.5 entanglements per chain are required in order for a polymer to form a continuous fibre during electrospinning (i.e. electrospinability) [43]. Hence, the present experimental findings are not consistent with the view that fibre 
formation during electrospinning is inextricably linked to intermolecular chain entanglements [24, 43-45].

For example, when food grade polysaccharides were electrospun [15], it was found that highest Trouton ratios (the ratio of extensional viscosity to shear viscosity) corresponded to better electrospinability. This suggests that rheological elasticity (storage modulus $>$ loss modulus) is an important property for the electrospinability of polysaccharides.

\section{Experimental procedures}

\subsection{Materials}

A culinary grade glucose syrup was used as received for the electrospinning experiments (Queen Fine Foods Ltd., Brisbane, Australia). The syrup is a glucose extract derived from corn that contains traces of sulfur dioxide (preservative 220) and sodium sulphite salts (80$150 \mathrm{mg} / \mathrm{kg} \max$ ). Mass spectrometry and high performance liquid chromatography confirmed that the syrup was mainly composed of monosaccharide (glucose and fructose $\left(\mathrm{C}_{6} \mathrm{H}_{12} \mathrm{O}_{6}\right)$ ), disaccharide (sucrose and maltose $\left(\mathrm{C}_{12} \mathrm{H}_{22} \mathrm{O}_{11}\right)$ ), and trisaccharide (raffinose $\left(\mathrm{C}_{18} \mathrm{H}_{32} \mathrm{O}_{16}\right)$ ). Hence, a series of heterogeneous solutions using pure glucose $(99.5 \%)$, sucrose $(99.5 \%)$, fructose $(99 \%)$, maltose (99\%) and raffinose (98\%) were synthesized to test its electrospinability. Materials were used as received from Sigma, without any modification.

\subsection{Materials characterisation}

\subsubsection{High performance liquid chromatography}

Sample preparation was carried out by dissolving a weighed amount of syrup in $1 \mathrm{~mL}$ of reverse osmosispurified water [46]. After centrifugation at $14000 \mathrm{rev} / \mathrm{min}$ for $10 \mathrm{~min}$, the supernatant was placed in a HPLC vial. Retention times and response factors generated by calibration standards of known sugars were used to identify and calculate concentrations of the unknown components [47, 48]. The concentrations were compared against three different points of the calibration curves for each sugar, and the results obtained had a coefficient of determination $\left(\mathrm{r}^{2}\right)$ close to unity (0.999). HPLC analysis was carried out using a Waters 2690 Pump, auto-sampler and Econosphere 5 micron amino column at $30^{\circ} \mathrm{C}$. An isocratic mobile phase of $75.0 \%$ acetonitrile:water was used. Eluted sugars were detected using a Waters 2414 refractive index detector at $40^{\circ} \mathrm{C}$.

\subsubsection{Rheological properties}

The rheological behaviour of the syrup was measured using an Anton-Paar MCR series rheometer (Anton Paar $\mathrm{GmbH}$, Graz, Austria). All experiments were performed with a cone and plate geometry configuration $(50 \mathrm{~mm}$ diameter) at a constant temperature of $50^{\circ} \mathrm{C}$. The response of the syrup was measured in both rotational mode as a function of the shear rate (0.0001-100 s $\left.{ }^{-1}\right)$ and oscillatory mode as function of the angular frequency $\left(0.01-100 \mathrm{~s}^{-1}\right)$.

\subsubsection{Preparation and characterization of electrospinning solutions}

Solutions were prepared for electrospinning with a total saccharide concentration of $75 \mathrm{wt}$ \% by dissolving the saccharide in de-ionised water at $50^{\circ} \mathrm{C}$ on a water bath controlled by a magnetic stirrer (Table 2). This concentration was chosen to ensure saccharide saturation of the solutions for the given experimental conditions. Saccharide solutions were stored within sealed glass containers during their preparation to minimize evaporation. Subsequently, all solutions were stored at $50^{\circ} \mathrm{C}$ due to the tendency for the saccharides to partially precipitate from a saturated solution at ambient temperature. An aqueous 75 wt. \% raffinose solution exhibited precipitation at $50^{\circ} \mathrm{C}$, hindering the electrospinning of this solution. Thus, an aqueous 65 wt. \% raffinose solution (A3) was required to be prepared for the electrospinning experiments. All of the physical properties of the solutions were measured at $50^{\circ} \mathrm{C}$ to avoid precipitation of the saccharides. The $\mathrm{pH}$ of the sac-

Table 1: Aqueous saccharide solutions and designations.

\begin{tabular}{lll}
\hline Composition & Concentration & $\begin{array}{l}\text { Sample } \\
\text { code }\end{array}$ \\
\hline Glucose & 75 wt. \% & A1 \\
Fructose & 75 wt. \% & B1 \\
Glucose/fructose & 37.5 wt. \% of each & C1 \\
Sucrose & 75 wt. \% & A2 \\
Maltose & 75 wt. \% & B2 \\
Sucrose/maltose & 37.5 wt. \% of each & C2 \\
Raffinose & 65 wt. \% & A3 \\
Raffinose/sucrose/maltose & 25 wt. \% of each & B3 \\
Raffinose/glucose/fructose & 25 wt. \% of each & C3 \\
\hline
\end{tabular}




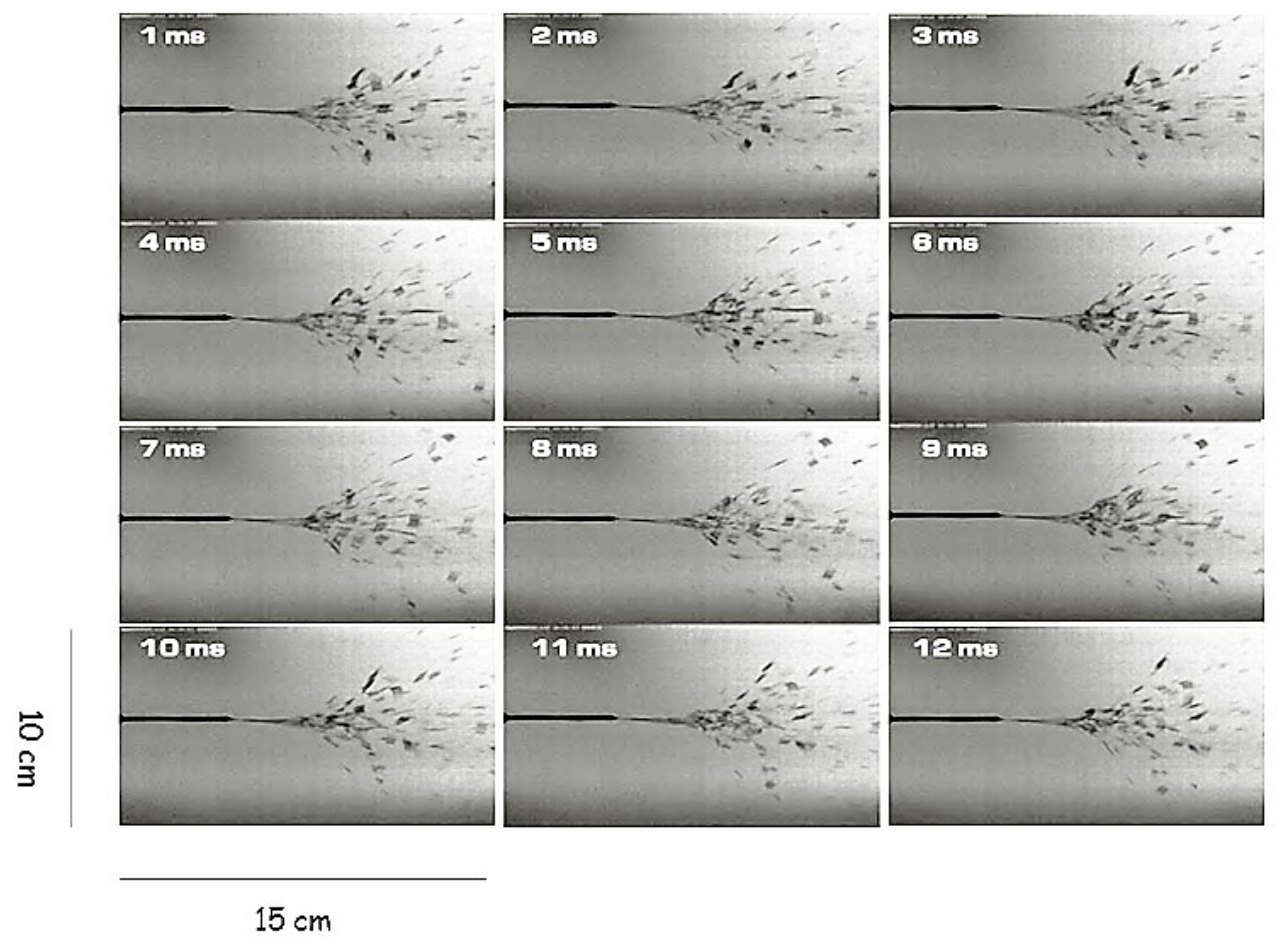

Figure 1: Series of high speed photographs taken at $1000 \mathrm{fps}$ during the electrospinning of the glucose syrup.

charide solutions was measured using a $\mathrm{pH}$ meter (SevenEasy, Mettler-Toledo GmbH, Greifensee, Switzerland). The surface tension was measured using a goniometer KSV CAM200 (KSV Instruments Ltd., Finland).

The electrical conductivity of the saccharide solutions was measured using a conductivity meter (EDT Instruments, RE387TX, Dover, United Kingdom). The pH, electrical conductivity and surface tension of the de-ionised water used in this work were $6.89,8 \mu \mathrm{S} / \mathrm{cm}$ and $78 \mathrm{mM} / \mathrm{m}$, respectively. All reported values are the averaged numbers based on three replicate measurements.

\subsubsection{Electrospinning process and characterization of electrospun materials}

A syringe pump (NE-500, New Era Pump Systems Inc., NY, USA) was used to deliver the electrospinning solution to the spinneret (metal hypodermic syringe needle, internal diameter of $0.3 \mathrm{~mm}$ ) at a flow rate of $0.5 \mu \mathrm{l} / \mathrm{min}$. All solutions were supplied to the spinneret, through a temperature controlled electric glass syringe cover, at a temperature of $50^{\circ} \mathrm{C}\left( \pm 2^{\circ} \mathrm{C}\right)$, to avoid precipitation of the saccharides from solution. Electrospun samples were collected using a grounded aluminium foil substrate. The electrospinning apparatus was enclosed in a grounded Faraday cage. Electrospinning of the various solutions was performed using an applied voltage of $+10 \mathrm{kV}$ and a spinneretto-collector distance of $15 \mathrm{~cm}(0.66 \pm 0.03 \mathrm{kV} / \mathrm{cm})$. The temperature and relative humidity within the set up were maintained at $30 \pm 1^{\circ} \mathrm{C}$ and $35 \pm 3 \%$, respectively. The elevated ambient temperature was generated by the halogen lamps required for the high-speed photography. Electrospun samples were stored in a temperature-controlled desiccator at low ambient humidity prior to characterisation. A high-speed motion camera (MotionPro ${ }^{\circledR}$ X3) with sensitivity of $1280 \times 1024$ pixels was used for capturing photographic images of the jet during flight. The fibre in flight was illuminated by $6 \times 12 \mathrm{~V} / 50 \mathrm{~W}$ halogen lamps to provide consistent lighting conditions during video capture.

\subsubsection{Microstructural analysis}

The microstructure of electrospun samples was examined with scanning electron microscopy (JCM-5000 NeoScope, JEOL Ltd., Tokyo, Japan). Samples were directly observed without a conductive coating being necessary during imaging with secondary electrons at an accelerating voltage of $10 \mathrm{kV}$ in high vacuum mode. 
Table 2: Physical properties of the glucose syrup at $25^{\circ} \mathrm{C}$.

\begin{tabular}{ll}
\hline Property & Value \\
\hline Concentration & $75 \%$ wt. \% syrup $/ \mathrm{H}_{2} \mathrm{O}$ \\
Surface tension & $98.11 \mathrm{mN} / \mathrm{m}$ \\
Conductivity & $1.5 \mu \mathrm{S} / \mathrm{cm}$ \\
$\mathrm{pH}$ & 5.5 \\
Density & $1.5 \mathrm{~g} / \mathrm{ml}$ \\
Zero shear viscosity & $38,600 \mathrm{~Pa} \cdot \mathrm{s}$ \\
\hline
\end{tabular}

\section{Results and discussion}

\subsection{Glucose syrup properties}

Typical electrospinning behaviour was observed for the glucose syrup, consisting of stable and chaotic jet formation (see Figure 1). These photographs show the heaviest parts of the electrospun jet as dark beads, within the spinning cone. The polarity of the applied voltage did not influence the electrospinning behaviour of the glucose syrup, hence positive polarity was used throughout the experimentation. The glucose syrup was reduced to $75 \%$ wt. concentration, however its viscosity remained considerable high regardless of the dilution, see (Table 2).

\subsubsection{Glucose syrup processing}

These results show for the first time that the glucose syrup, which actually contained a mixture of mono-, di- and trisaccharides, could form continuous filaments during electrospinning. The highly beaded structures of the filaments (see Figure 2C and 2D) clearly suggest a viscoelastic behaviour of the material during electrospinning. However, the high hydrophilicity of saccharides, does not promote the stabilization of its fibrous structure for long periods of time, hence its flattened geometry, as shown in Figure 2A and 2B.

\subsection{Glucose syrup characterization}

\subsubsection{Scanning Electron Microscopy}

The syrup was analysed by mass spectrometry (MS) and high performance liquid chromatography (HPLC) to confirm the absence of starch or other polymeric materials or other long chain polymeric materials that could be responsible for fibre formation. However, HPLC analysis indicated the presence of only the monosaccharides glucose
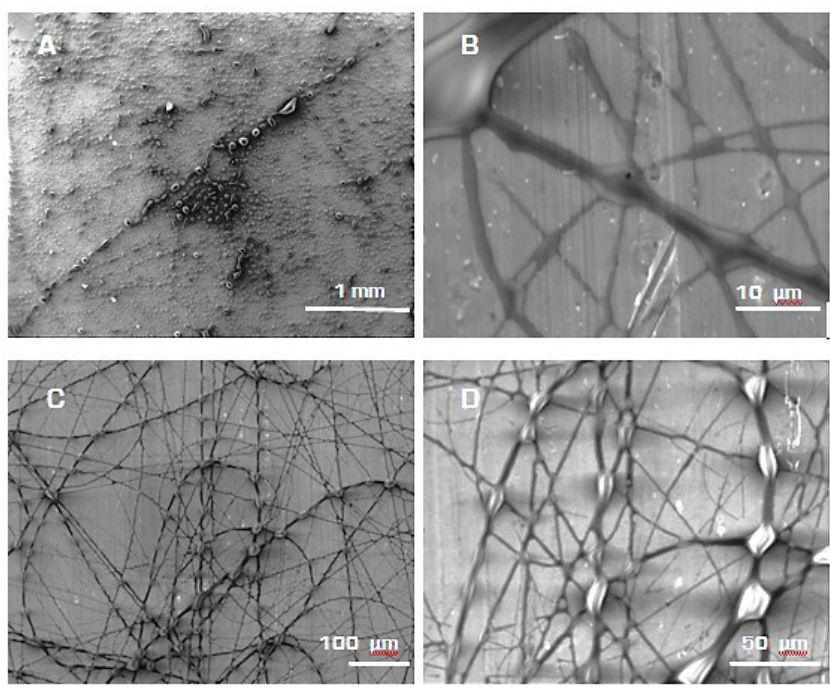

Figure 2: Four different samples of the electrospun glucose syrup at varying magnifications; as seen by the Scanning electron microscope. Area samples for the SEM analysis were chosen at random.

and fructose, the disaccharide sucrose, and the trisaccharide raffinose, but no larger oligo- or polymeric structures (see Figure 4). Furthermore, MS confirmed the presence of these materials but provided no evidence of larger polymeric structures in the syrup (see Figure 3).

\subsubsection{Mass - spectrometry}

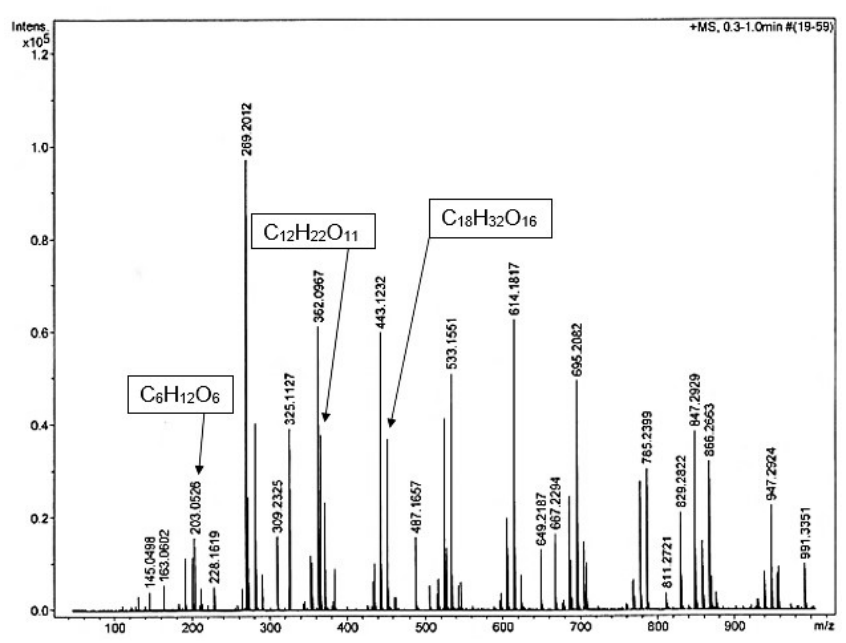

Figure 3: Mass spectrum from MS showing the intensity (y-axis) as a function of the mass-to-charge ratio (x-axis). The presence of the isomers of glucose, sucrose and raffinose are indicated at mass-tocharge ratios of 203.05, 365.1 and 527.16, respectively. 


\subsubsection{High Performance Liquid Chromatography}

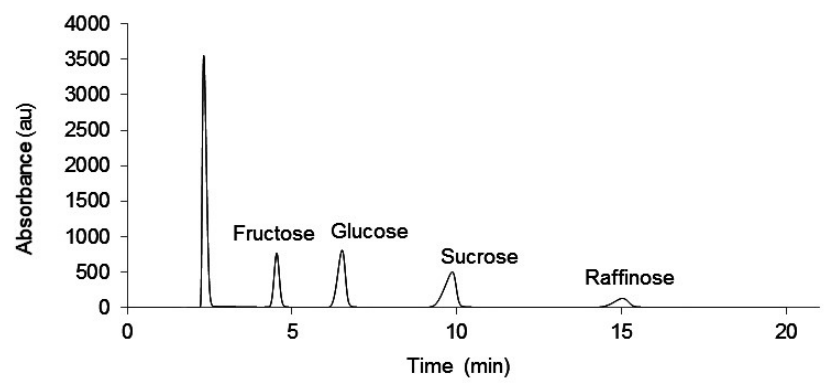

Figure 4: HPLC chromatogram confirming the presence of fructose, glucose, sucrose and raffinose in the glucose syrup. The first peak is the injection peak.

\subsubsection{Rheometry}

Visco-elasto capillary theory sets different boundaries for spinnability depending on rheological properties as described by several non-dimensional numbers such as the Deborah number (De) and the Ohnesorge number (Oh), which should comply with the following electrospinnability condition $\mathrm{De} \geq \mathrm{Oh} \geq 1$ [49]. The Deborah number is indicative of the relaxation time of the material under an applied stress a given stress and the Ohnesorge number indicating the viscous response of a material to a given stress (or the inverse of a Reynolds number based on a characteristic capillary velocity) [49]. For Deborah numbers below unity, elastic effects do not stabilize the jet and Newtonianlike breakup dynamics are observed. For Ohnesorge numbers below unity, capillary wavelength instabilities are observed in the form of beaded filaments. However, the rheology results suggested that the glucose syrup performs as a polymer when mechanically stressed, since it showed a visco-elastic behaviour (see Figure 5A and 5B).

This apparent "viscoelasticity" agrees in principle with the visco-elasto-capillary thinning theory for complex fluids as described by McKinley [49]. Since the shear thinning response of the viscosity measurements (Figure $5 \mathrm{~A}$ ), indicates the initial viscous response of the solution to an increasing shear rate, followed by an elastic thinning response as indicated by a decreasing shear viscosity (slope) beyond a shear rate of $\sim 0.01 \mathrm{~s}^{-1}[50,51]$. However, the frequency response of the storage and loss modulus of the syrup shows that this behaviour is mostly elastic [52], since the storage modulus is greater than the loss modulus over the entire frequency range measured

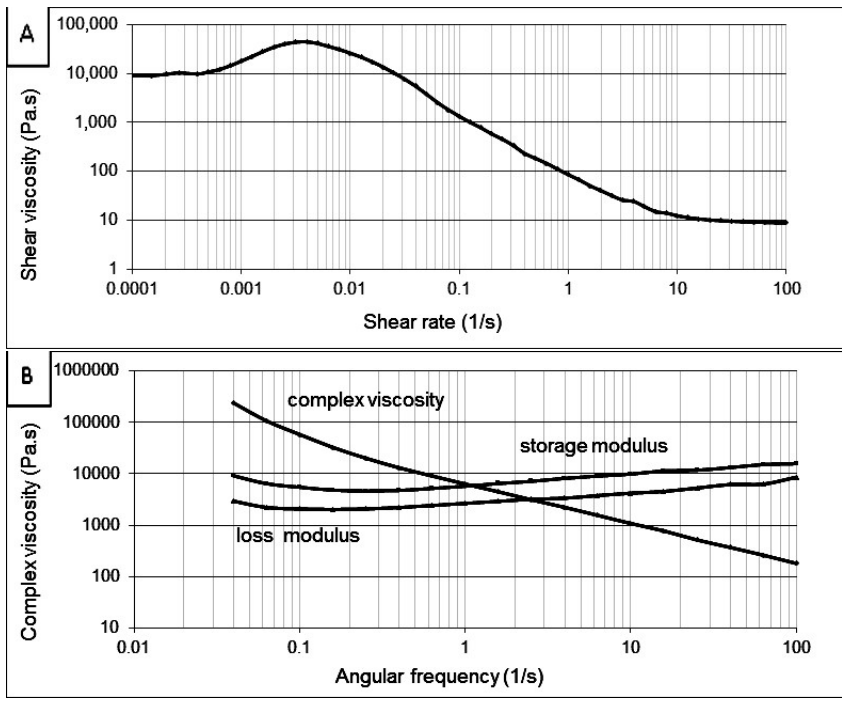

Figure 5: (A) shows the shear viscosity as a function of shear rate, (B) shows the complex viscosity, loss and storage modulus as a function of angular frequency of the glucose syrup.

(Figure 5B). This further supports the evidence that higher Trouton ratios corresponded to better electrospinability (storage modulus > loss modulus) [15].

\subsection{Saccharide solutions characterization}

\subsubsection{Microstructural Analysis (SEM)}

It is evident from Figures 6 and 7 that neither the monosaccharides glucose or fructose, nor the trisaccharide raffi-
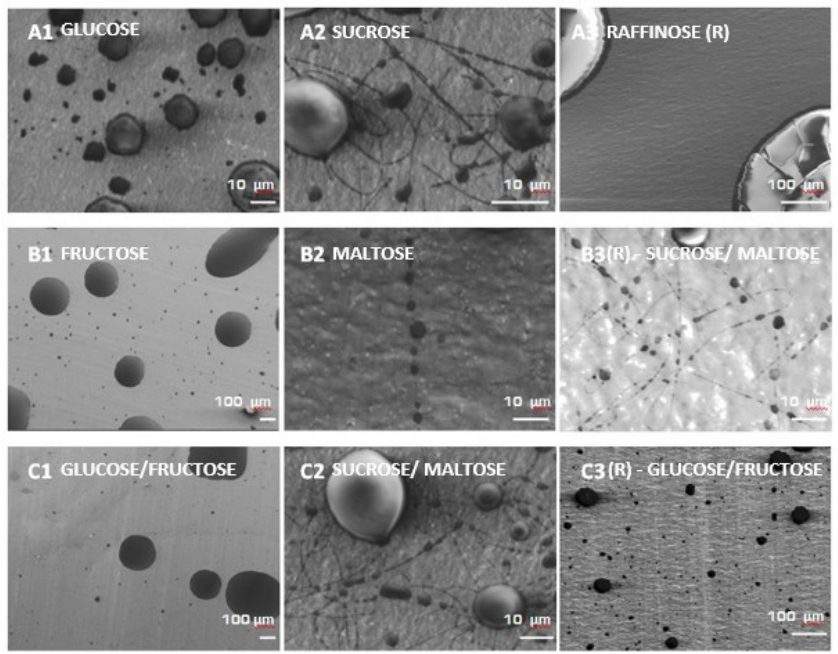

Figure 6: Representative scanning electron micrographs of the electrospun solutions. Images are presented at different magnifications to aid structure visualization. 
nose, nor their combinations, were able to form fibres via the electrospinning process. In contrast, the disaccharides sucrose and maltose, and combinations of them, gave more continuous filament formation (see Figure 6 - A2, C2 \& B3). For solutions series $1(\mathrm{ABC})$ as well as for $\mathrm{A} 3$ and $\mathrm{C} 3$, no electrospinning or electrospun fibre formation was observed, rather only an emission of droplets (electrospray).

\subsubsection{High speed photography and microstructural Analysis}
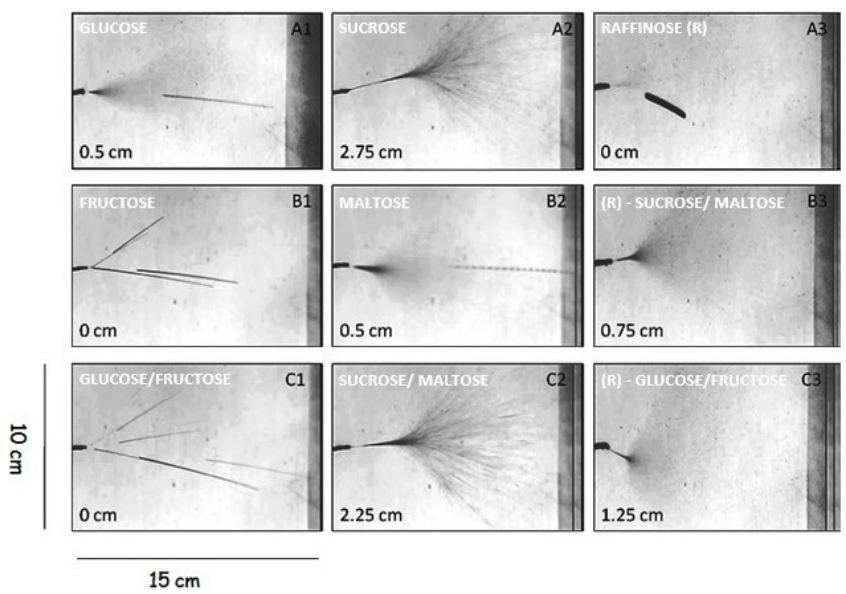

Figure 7: Representative photographs of the electrospinning behaviour of each solution showing the length of the stable jet.

\subsubsection{Saccharide solutions properties}

Figures 8 and 9 show that sucrose (A2), the material with better electrospinnability - highest amount of continuous filament formation, had the lowest conductivity and the highest $\mathrm{pH}$. This observation seems counter-intuitive according to some reports on polymer electrospinnability [53-55], wherein the ionic conductivity can be an important parameter for the electrospinnability of polymers, since an increment in the conductivity relates to an increment in the amount or capacity of the charge carriers (ions), and therefore better charge mobility or conductivity [56-59].

However, the fact that sucrose had the lowest surface tension is in agreement with visco-elasto capillary theory, which states that an electrospinnable system will tend to minimise its total boundary energy (effectively its surface tension) to form continuous filaments $[43,45,60]$.

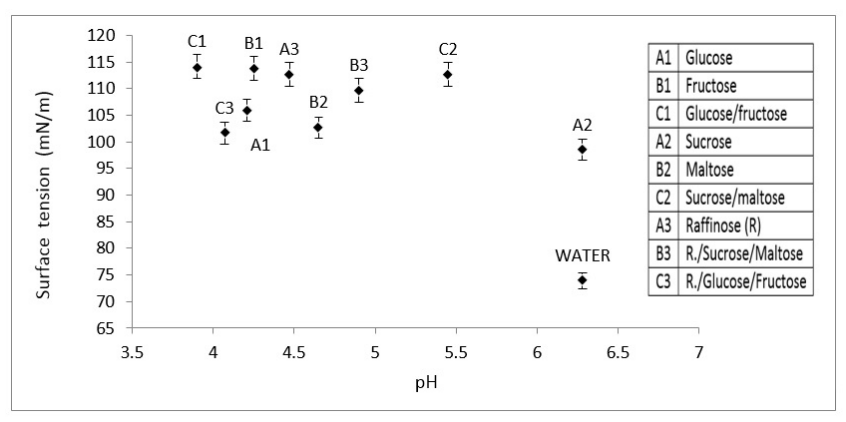

Figure 8: Surface tension as a function of $\mathrm{pH}$. All measurements were taken at $50^{\circ} \mathrm{C} \pm 3.5^{\circ} \mathrm{C}$.

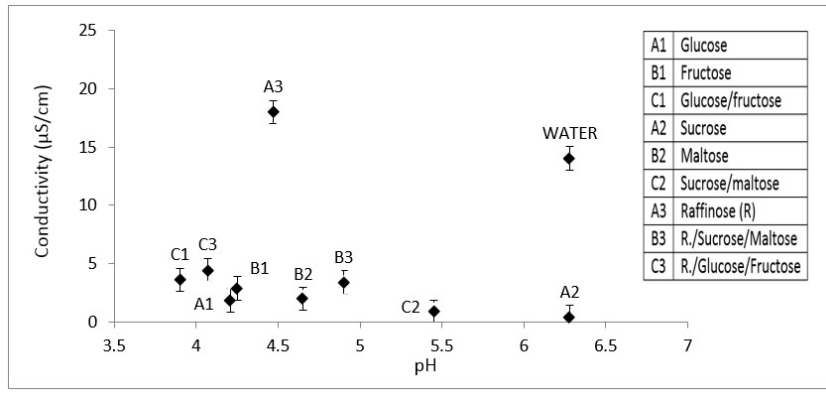

Figure 9: Electrical conductivity as a function of $\mathrm{pH}$. All measurements were taken at $50^{\circ} \mathrm{C} \pm 3.5^{\circ} \mathrm{C}$.

Nonetheless, it is not clear how intermolecular hydrogen bonding systems, for example concentrated saccharide solutions, could play a role in the cohesive surface energy during electrospinning $[61,62]$.

\subsection{Syrup replica}

To further confirm the electrospinnability of the commercially supplied glucose syrup, a duplicate solution was made up by combining the pure components that had been previously identified by HPLC, at similar relative concentrations of each material. Namely glucose (34 wt. \%), fructose (22.5 wt. \%), sucrose (32 wt. \%), and raffinose (11.5 wt. \%) in distilled water, to give a final total solids concentration of 85 wt. \%.

\subsubsection{Syrup replica - microstructural analysis (SEM)}

The fact that this solution exhibited similar behaviour to the commercially supplied glucose syrup (i.e. produced continuous filamentation), provided strong evidence that the presence of polymeric materials, which might have been present but were not detected by HPLC or MS, was 

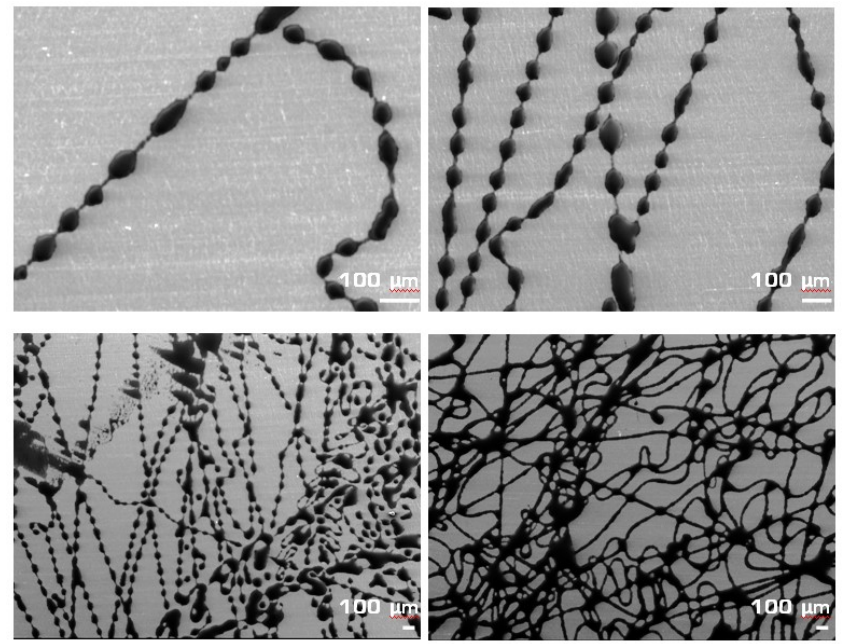

Figure 10: Scanning Electron Microscope (SEM) images of fibres made from the duplicate of the glucose syrup.

not a requirement for electrospinability. The observations of this study therefore do not fit into the theoretical framework of chain entanglement [43] and suggest that alternative mechanisms are involved in the production of saccharide electrospun fibres.

\section{Conclusions}

These results show for the first time that a heterogeneous mixture containing mono-, di- and tri- saccharides can form continuous filaments during electrospinning, and can exhibit the same behaviour as polymeric solutions whilst being electrospun; namely the formation of a stable jet followed by whipping instability.

From the rheology analysis it is suggested that storage modulus > loss modulus could be the cause for the apparent elasticity of the material, allowing for the unusual stretching of the stable electrospun jet. Furthermore, the surface tension data for sucrose agrees with elastocapillary theory i.e. that "an electrospinnable system will tend to minimize its superficial energy to form continuous filaments" [49, 52, 56, 63]. Remarkably, the electrospinnability of all these materials is not consistent with current proposed chain entanglement [43] theory of electrospinning.

Conclusively, chain entanglement theory does not adequately describes the electrospinning behaviour of saccharides, which exhibited non-linear viscoelasticity and complex charge transfer mechanisms due to their extensive intermolecular bonding [42].
Acknowledgement: We thank the National Institute of Science and Technology of Mexico (CONACYT) and the New Zealand Science Foundation for financial support. Support from the NZ Institute for Plant and Food Research Ltd, Electrospinz Ltd, and Revolution Fibres Ltd., and Deborah LeCorre is also gratefully acknowledged.

\section{References}

[1] Reneker D.H., Yarin A.L., Electrospinning jets and polymer nanofibers, Polymer 2008, 49(10): p. 2387-2425.

[2] Doshi J., Reneker D.H., Electrospinning Process and Applications of Electrospun Fibers, Journal of Electrostatics 1995, 35: p. 151-160.

[3] Andreas Greiner J.H.W., Electrospinning: A Fascinating Method for the Preparation of Ultrathin Fibers, Angewandte Chemie 2007, 46: p. 5670-5703.

[4] Spivak A.F., Dzenis Y.A., Reneker D.H., A Model of Steady State Jet in the Electrospinning Process, Mechanics Research Communications 2000, 27(1): p. 37-42.

[5] Martino A.D., Sittinger M., Risbud M.V., Chitosan: A versatile biopolymer for orthopaedic tissue-engineering, Biomaterials 2005, 26: p. 5983-5990.

[6] Ohkawa K. et al., Electrospinning of chitosan, Macromolecular Rapid Communications 2004, 25: p. 1600-1605.

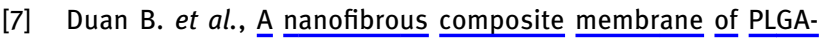
chitosan/PVA prepared by electrospinning, European Polymer Journal 2006, 42(9): p. 2013-2022.

[8] Ignatova M., Manolova N., Rashkov I., Novel antibacterial fibers of quaternized chitosan and poly(vinyl pyrrolidone) prepared by electrospinning, European Polymer Journal 2007, 43: p. 11121122.

[9] Lin T. et al., Using chitosan as a thickener for electrospinning dilute PVA solutions to improve fibre uniformity, Nanotechnology 2006, 17(15): p. 3718-3723.

[10] Bhattarai N. et al., Electrospun chitosan-based nanofibers and their cellular compatibility, Biomaterials 2005, 26: p. 61766184.

[11] Frey M.W., Electrospinning cellulose and cellulose derivates, Polymer Reviews 2008, 48(2): p. 378-391.

[12] Han S.O. et al., Electrospinning of cellulose acetate nanofibres $\underline{\text { using a mixed solvent of acetic acid-water: Effects }} \underline{\text { of solvent }}$ composition on the fiber diameter, Materials Letters 2008,62 : p. 759-762.

[13] Chen L. et al., Electrospun cellulose acetate fibers containing chlorhexidine as a bactericide, Polymer 2008, 49(5): p. 12661275.

[14] Liu H., Hsieh Y.L., Ultrafine fibrous cellulose membranes from electrospinning of cellulose acetate, Journal of Polymer Science 2002, 40: p. 2119-2129.

[15] Wang Y. et al., Composite electrospun nanomembranes of fish scale collagen peptides/chito-oligosaccharides: Antibacterial properties and potential for wound dressing, International Journal of Nanomedicine 2011, 6: p. 667-676.

[16] Dong B. et al., Electrospinning of collagen nanofiber scaffolds from benign solvents, Macromolecular Rapid Communications 
2009, 30: p. 539-542.

[17] Chen Z. et al., Intermolecular interactions in electrospun collagen-chitosan complex nanofibers, Carbohydrate Polymers 2008, 72: p. 410-418.

[18] Hofman K. et al., Effects of the molecular format of collagen on characteristics of electrospun fibres, Journal of Materials Science 2012, 47(3): p. 1148-1155.

[19] Yao C., Li X.S., Song T.Y., Electrospinning and crossfinking of Zein nanofiber mats, Journal of Applied Polymer Science 2007, 103(1): p. 380-385.

[20] Selling G.W. et al., Electrospun zein fibers using glutaraldehyde as the crosslinking reagent: Effect of time and temperature, Macromolecular Chemistry and Physics 2008, 209: p. 10031011.

[21] Selling G.W. et al., Impact of solvent on electrospinning of zein and analysis of resulting fibers, Macromolecular Chemistry and Physics 2007, 208: p. 1002-1010.

[22] Miyoshi T., Toyohara K., Minematsu H., Preparation of ultrafine fibrous zein membranes via electrospinning, Polymer International 2005, 54: p. 1187-1190.

[23] Jiang H.L., Zhao P.C., Zhu K.J., Fabrication and characterization of zein-based nanofibrous scaffolds by an electrospinning method, Macromolecular Bioscience 2007, 7(4): p. 517-525.

[24] Woerdeman D.L. et al., Electrospun fibers from wheat protein: Investigation of the interplay between molecular structure and the fluid dynamics of the electrospinning process, Biomacromolecules 2005, 6(2): p. 707-712.

[25] Kowalczyk T. et al., Electrospinning of bovine serum albumin. Optimization and the use for production of biosensors, Biomacromolecules 2008, 9: p. 2087-2090.

[26] Wnek G.E. et al., Electrospinning of Nanofiber Fibrinogen Structures, Nano Letters 2003, 3(2): p. 213-216.

[27] Dror Y. et al., Nanofibers made of globular proteins, Biomacromolecules 2008, 9: p. 2749-2754.

[28] Huang Z.-M. et al., Electrospinning and mechanical characterisation of gelatin nanofibers, Polymer 2004, 45: p. 5361-5368.

[29] Zhang Y. et al., Electrospinning of gelatin fibers and gelatin/PCL composite fibrous scaffolds, Journal of Biomedical Materials Research 2004: p. 156-165.

[30] Mo X.M. et al., Electrospun P(LLA-CL) nanofiber: A biomimetic extracellular matrix for smooth muscle cell and endothelial cell proliferation, Biomaterials 2004, 25: p. 1883-1890.

[31] Stijnman A.C., Bodnar I., Tromp R.H., Electrospinning of foodgrade polysaccharides, Food Hydrocolloids 2011, 25(5): p. 1393-1398.

[32] Toskasa G. et al., Nanofibers based on polysaccharides from the green seaweed Ulva Rigida, Carbohydrate Polymers 2011, 84: p. 1093-1102.

[33] Boland E.D. et al., Electrospinning of Biopolymers (Natural and Synthetic) for Tissue Engineering Scaffolds, Polymer Preprints 2003, 44(2): p. 92-93.

[34] Li M. et al., Electrospun protein fibers as materials for tissue engineering, Biomaterials 2005, 26: p. 5999-6008.

[35] Schiffman J.D., Schauer C.L., A review: Electrospinning of biopolymer nanofibers and their applications, Polymer Reviews 2008, 48: p. 317-352.

[36] Duling R.R. et al., Mechanical characterization of electrospun polycaprolactone $(\mathrm{PCL})$ : A potential scaffold for tissue engineering, Journal of Biomechanical Engineering 2008, 130(1): p. 011006.
[37] Kidoaki S., Kwon I.K., Matsuda T., Mesoscopic spatial designs of nano- and microfiber meshes for tissue-engineering matrix and scaffold based on newly devised multilayering and mixing electrospinning techniques, Biomaterials 2005, 26(1): p. 37-46.

[38] Lee K.Y. et al., Electrospinning of polysaccharides for regenerative medicine, Advanced Drug Delivery Reviews 2010, 61: p. $1020-1032$

[39] Garg H.G., Cowman M.K., Hales C.A., Carbohydrate chemistry, biology and medical applications, 2008, Oxford, U.K.: Elsevier, 414.

[40] Boddohi S., Kipper M.J., Engineering nanoassemblies of polysaccharides, Advanced Materials 2010, 22: p. 2998-3016.

[41] Manandhar S., Vidhate S., D’Souza N., Water soluble levan polysaccharide biopolymer electrospun fibers, Carbohydrate Polymers 2009, 78: p. 794-798.

[42] Hermida-Merino D. et al., Electrospun supramolecular polymer fibres, European Polymer Journal 2012, 48: p. 1249-1255.

[43] Shenoy S.L. et al., Role of chain entanglements on fiber formation during electrospinning of polymer solutions: Good solvent, non-specific polymer-polymer interaction limit, Polymer 2005, 46: p. 3372-3384.

[44] Shenoy S.L., Bates W.D., Wnek G., Correlations between electrospinnability and physical gelation, Polymer 2005, 46(21): p. 8990-9004.

[45] Woerdeman D.L., Shenoy S., Breger D., Role of chain entanglements in the electrospinning of wheat protein-poly(vinyl alcohol) blends, Journal of Adhesion 2007, 83(8): p. 785-798.

[46] Neue U.D., HPLC columns: Theory, technology and practice, ed. M. Zoubair El Fallah, 1997, New York, NY: John Wiley and Sons, 393.

[47] Lopez Hernandez J.L. et al., High-performance liquid chromatographic determination of mono- and oligosaccharides in vegetables with evaporative light-scattering detection and refractive index detection, Journal of Chromatographic Science 1998, 36: p. 293-298.

[48] Basumallick L., Hurum D., Rohrer J., Methods for determining sugars and hydroxymethylfurfural in biomass, 2011, Thermo Fisher Scientific: Sunnyvale, CA. p. 1-6.

[49] McKinley G.H., Visco-elasto-capillary thinning and break up of complex fluids. HML Report Number 05-P-04, 2005, Massachusetts Institute of Technology Cambridge, MA. p. 49.

[50] Navier C.L., Memoire sur les lois du mouvement des fluides, Memoires de la Academie des Sciences 1822, 6: p. 389-440.

[51] Baidakov V.G. et al., The van der Waals theory of capillarity and computer simulation, Colloid Journal 2001, 64(6): p. 661-670.

[52] Doyle P.S. et al., Relaxation of dilute polymer solutions following extensional flow, Journal of Non-Newtonian Fluid Mechanics 1998, 76: p. 79-110.

[53] Reneker D.H. et al., Bending instability of electrically charged liquid jets of polymer solutions in electrospinning, Journal of Applied Physics 2000, 87(9): p. 4531-4547.

[54] Thompson C.J. et al., Effects of parameters on nanofiber diameter determined from electrospinning model, Polymer 2007, 48: p. 6913-6922.

[55] Han T., Yarin A.L., Reneker D.H., Viscoelastic electrospun jets: Initial stresses and elongational rheometry, Polymer 2008, 49: p. 1651-1658.

[56] Lukas D. et al., Physical principles of electrospinning: Electrospinning as a nano-scale technology of the twenty-first century, Textile Progress 2009, 41(2): p. 59-140. 
[57] Ramakrishna S. et al., An introduction to electrospinning and nanofibers, 2005, Singapore: World Scientific Publishing, 382.

[58] Reneker D.H., Fong H., Polymeric nanofibers, 2006, Washington D.C.: American Chemical Society, 464.

[59] Andrady A.L., Science and technology of polymer nanofibers, 2008, Hoboken, NJ: John Wiley and Sons, 420.

[60] Nie H. et al., Effects of chain conformation and entanglement on the electrospinning of pure alginate, Biomacromolecules 2008, 9: p. 1362-1365.

[61] Fabri D., Williams M.A.K., Halstead T.K., Water T2 relaxation in sugar solutions, Carbohydrate Research 2005, 340: p. 889905.
[62] Arikawa T., Nagai M., Tanaka K., Characterizing hydration state in solution using terahertz time-domain attenuated total reflection spectroscopy, Chemical Physics Letters 2008, 457: p. 1217.

[63] McKinley G.H., Dimensionless groups for understanding free surface flows of complex fluids. HML Report Number 05-P-05, 2005, Massachussetts Institute of Technology: Cambridge, MA. p. 9. 